Journal of Engineering and Applied Sciences 14 (1): 211-218, 2019

ISSN: 1816-949X

(C) Medwell Journals, 2019

\title{
Traffic Surveillance System for Vehicles Counting and Speed Estimation
}

\author{
Laith A. H. Al-Shimaysawee, Ali H. A. Aldabbagh and Hussein M. H. Al-Rikabi \\ Robotics and Computer Vision Research Group (RCVRG), \\ Department of Electrical Engineering, Faculty of Engineering, University of Kufa, Najaf, Iraq
}

\begin{abstract}
In this study, we present an algorithm to count vehicles and estimate their speed using cameras mounted on roads. The first step in the proposed algorithm is motion detection and then vehicles are detected after some threshold, morphological and contour operations. After detection process, vehicles can be counted and their speed can be measured. The vehicles counting part of the proposed algorithm was tested using 6 data sets which show different times and weather conditions and compared with the manual counting which is considered as ground truth. The vehicle speed measurement part of the proposed system was tested using 5 videos from five datasets available online. These data sets have a ground truth file for the actual speed of each vehicle. The actual speed was recorded by a high precision speed meter. The measured speeds were compared with the recorded ground truth values. Both parts of our system showed very good results. The overall counting accuracy was $(90.8 \%)$ and the accuracy of speeds measurements that is within the acceptable error interval was $(88.21 \%)$. The speed measurement was considered acceptable if it is within the $(-5,+5 \mathrm{~km} / \mathrm{h})$.
\end{abstract}

Key words: Vehicles counting, speed measurement, traffic surveillance, motion detection, vehicle detection, good results

\section{INTRODUCTION}

In recent years, many artificial intelligence and computer vision technologies have been developed significantly in different aspects and various applications such as Drive Assist Systems (DAS) (McCall and Trivedi, 2006), surveillance systems (Wang, 2012), computer and robot interactive applications (Fujii et al, 2018), rescue assist and military applications (Matsuno and Tadokoro, 2004) and many others (Fuchs et al., 1998; Taylor et al., 2016). It is a very important to develop such techniques to monitor traffic in cities in terms of collecting information about the roads that have traffic issues and the time of traffic and then using this collected information to develop the road ways and maybe establish new ones to solve the traffic problems.

Moreover, some artificial intelligence techniques can be combined with traffic light system to produce an intelligent traffic light that provides more time to empty the crowded sides rather than providing time equally to all direction regardless whether the road side is empty or crowded. On the other hand, some computer vision techniques can be developed to monitor the roads and streets in any city and help to locate any stolen vehicles and send their locations to the police station. In addition, such algorithms can be used to capture any vehicle that exceeds the speed limit and record its license plate to provide a fine to the driver. Using such technology is very efficient to solve many issues in the traffic surveillance and makes the research of the traffic police easier and effective. However, many developments are required to be done to improve the measurement accuracy and efficiency. In this study, we develop a camera surveillance system and propose a computer vision algorithm to count vehicles and estimate their speed. Using a camera to count vehicles and measure their speed is very beneficial if compared with Doppler and Radars, since, the camera surveillance system requires less cost and does not require frequent maintenance.

Literature review: In the following two subsections, we present a brief survey about the related research that have been proposed for vehicles counting and speed estimation using camera monitoring system.

Vehicles counting: A morphological operator was used for background registration technique and segmentation in Daigavane and Bajaj (2010). In this research, video camera system mounted on highways has been proposed to detect and count objects dynamically. Target objects were identified in the presence of partial occlusions and ambiguous poses by combining time domain statistical

Corresponding Author: Laith A. H. Al-Shimaysawee, Robotics and Computer Vision Research Group (RCVRG), Department of Electrical Engineering, Faculty of Engineering, University of Kufa, Najaf, Iraq 
measures and simple domain knowledge about these objects classes. The problems of unsupervised video/image segmentation and object modelling with multimedia inputs string were considered by Chen et al. (2001) for a real-life traffic monitoring for the purpose of capturing the spatial and temporal behaviors of the tracked vehicle objects. An enhanced particle filter for vehicle tracking was proposed by Gao et al. (2009) based on Redundant Discrete Wavelet Transform RDWT and Scale Invariant Feature Transform (SIFT) algorithms. To overcome the weakness of time domain for vehicle detection, a RDWT algorithm was used for detection purpose and then set of key points were extracted as particles and matched with the follow up image frames. Vehicle detection and classifications algorithms were proposed by Gupte et al. (2002) based on sequences of monocular images of the traffic scenes that are recorded by a still camera. The proposed algorithm by Gupte et al. (2002) consists of segmentation using current and prior frame difference, region tracking using spatial matching method, vehicle parameters recovery, vehicle identification, vehicle tracking and then finally vehicle classification. To overcome the challenges of classification of different vehicle classes such as sedans vs taxies, a rich edge representation using modified SIFT as descriptor based algorithm for vehicle classification was proposed by $\mathrm{Ma}$ and Grimson (2005). However, the algorithm fails for changes in view and occlusion. Buch et al. (2011), detection and classification of individual vehicles was done by combining $3 \mathrm{D}$ extended Histograms of Oriented Gradients (HOG). An image warping was used by Jun et al. (2008) and Salvi (2012) as a pre-processing step to generate a horizontal or vertical road segment to facilitate the detection and tracking. A cascaded regression model for vehicle counting and classification was proposed by Liang et al. (2015) which is able to deal with severe occlusions and a very low vehicle image resolution. Biswas et al. (2017), vehicle counting was done using the Gaussian background subtraction method. In addition, OverFeat framework is also proposed for vehicle counting in the earlier research. The OverFeat framework consists from machine learning classifier and Convolution Neural Network (CNN).

Speed estimation: There are many video based proposed algorithms for vehicle speed estimations based on background/foreground segmentation process for the purpose of motion detection. The segmentation process may be as simple as frame difference between the current and prior image frames such as Daniel and Pumrin (2000), Madasu and Hanmandlu (2010), Dogan et al. (2010) and
He and Yung (2007) or statistic models based on median such as Zhiwei et al. (2007), Maduro et al. (2008) and Palaio et al. (2009) or even using Gaussian distribution for segmentation purpose such as. Vehicle speed estimation is based on tracking image features or image regions. Tracking image regions based on direct blob analysis was proposed by Daniel and Pumrin (2000), Madasu and Hanmandlu (2010), He and Yung (2007), Maduro et al. (2008). In these proposed methods, moving blobs was created after filling the moving edges (Daniel Pumrin, 2000 ) or image patches or corners (Madasu and Hanmandlu, 2010) that resulted from morphological operators on binary images. However, these methods are very sensitive to shadow and illumination variations. In addition, the results of these algorithms are decent just in the case when a camera is mounted in high position above the road. Dogan et al. (2010), the researchers use LucasKanade optical flow algorithm to overcome the problems of blob analysis by directly tracking the distinctive features. However, the algorithm can only process single vehicle at a time, since, it deals with side view images. Vehicle speed estimation by tracking licence plate region was proposed by Garibotto et al. (2001), Czajewski and Iwanowski (2010) and Garg and Goel (2013). License palate recognition was done by Garibotto et al. (2001) using optical character recognition by template matching algorithm. The drawback of the research is that it depends on the OCR robustness and did not produce acceptable results even in a controlled environment. In addition, the algorithm proposed by Czajewski and Iwanowski (2010) also suffers from the same problem. To overcome the precise character segmentation problem, (Luvizon et al., 2017) proposed an algorithm for speed measurement which does not depend on character recognition but it tracks the selected features in license plate region.

Setup of system hardware: The hardware of the system consists of cameras fixed on high places such as bridges or traffic lights and lighting poles and mounted on the roads and streets for traffic surveillance and control. We fixed a camera on a pedestrian's bridge and mounted it on Kufa-Najaf main road, one of the main roads in Al-Najaf city. The camera that is used to record videos to test the proposed system was $16 \mathrm{MP}$ from Samsung Galaxy S5 mobile. The recorded data sets can be freely delivered for research purposes by request from the researchers. Figure 1 shows the system of the camera system with some parameters which are explained in the next study. Figure 1 is a modified sketch from (Luvizon et al. 2017). 


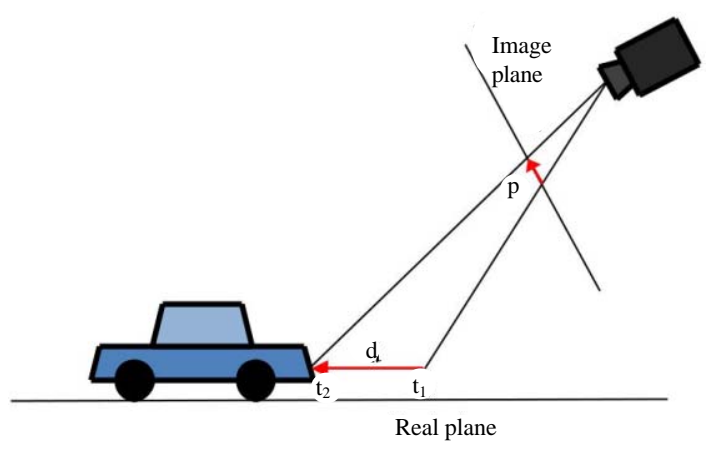

Fig. 1: Setup of the camera surveillance system

\section{MATERIALS AND METHODS}

Description of the proposed algorithm: The proposed approach has four main parts: motion detection, vehicles detection, counting vehicles and estimating vehicles speed. Figure 2 shows these main parts. In the first part (Fig. 2b), the process of detecting motion is established by subtracting two successive image frames Eq. 1 and then, if there is any motion, it will be detected. Fig. 3 shows motion detection process:

$$
\text { Detected-motion }=\text { Frame(i)-Frame(i-1) }
$$

In the second part (Fig. 2c), the first step in the vehicle detection process is to convert the coming image frame to grayscale image. Then, after a proper adjusted threshold, the image frame is converted to binary image and vehicles can be detected but with some noise appeared. By using morphological operations, the noise can be removed.

The structuring element used in the erosion operation was a square of side length of four pixels. The purpose of erosion operation is to remove noise. Then a dilation operation was used to combine the parts of expected vehicles to be detected and located. The structuring element used in the dilation operation was a square of side length of 10 pixels. The dilation operation has been repeated 5 times to ensure the separated parts of the expected vehicles are combined in one object. The values of structuring elements were selected based on many tests and experiments on the input videos. Then, the processed frame passes through a filter to keep only the objects with a size close to size range of the expected vehicles. The size range has been determined previously in the system hardware setup stage. Then, any object outside the region of interest is removed (Fig. 4 shows the region of interest). Figure 5 shows vehicles detection process.

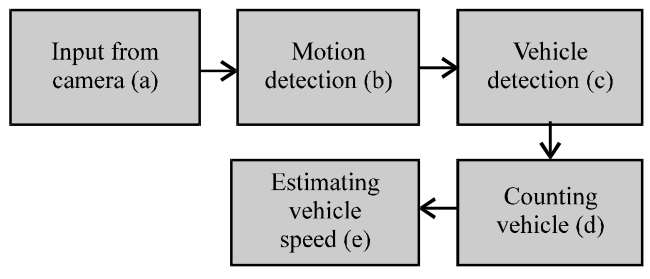

Fig. 2: Main parts of the proposed algorithm

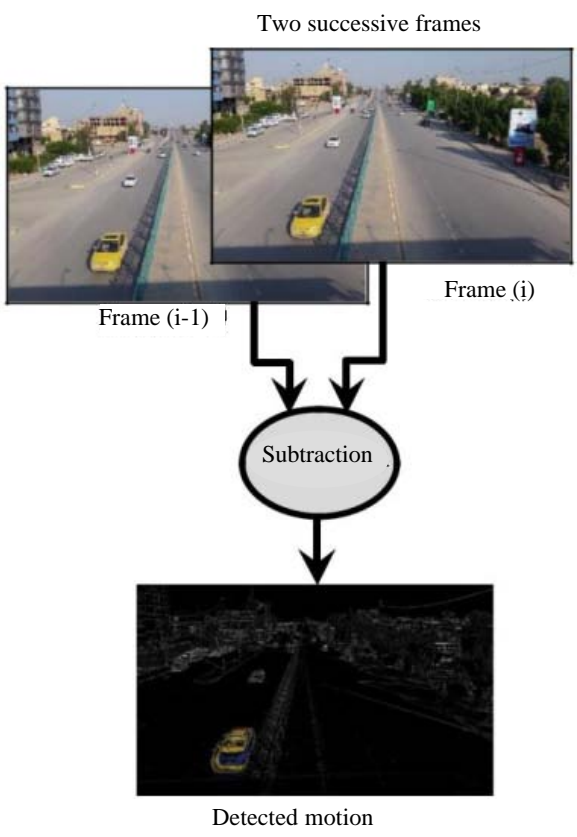

Fig. 3: Motion detection process

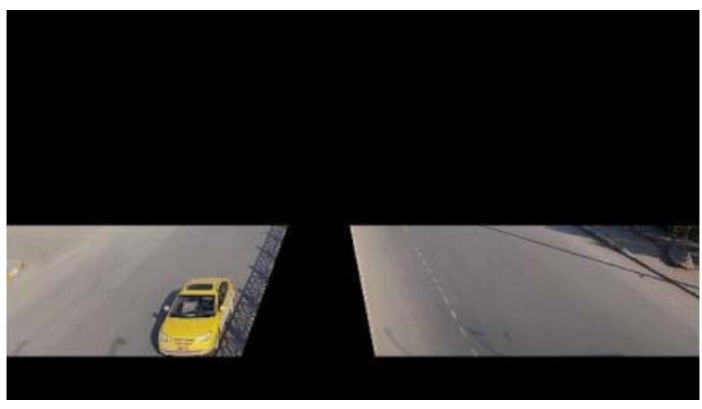

Fig. 4: Specified region of interests

In the third part (Fig. 2d), the algorithm counts the detected vehicles when pass a specified region of interest (Fig. 5). Figure 6 shows counting vehicles process.

In the last part (Fig. 2e), the speed of the detected vehicles can be estimated by the following procedure:

The speed can be measured using Eq. 2 by dividing the distance moved by the vehicle by the elapsed time for that move: 

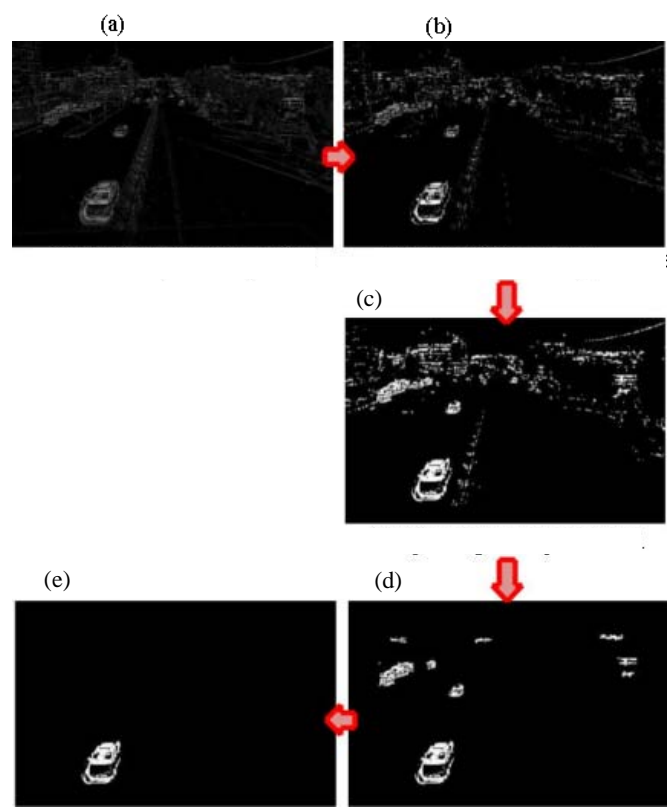

Fig. 5: Vehicles detection process: a) Converting to Grayscale; b) Threshold the image; c) Morphological operations; d) Detecting objects with size close to the expected vehicles sizes and e) Processing the specified region of interests

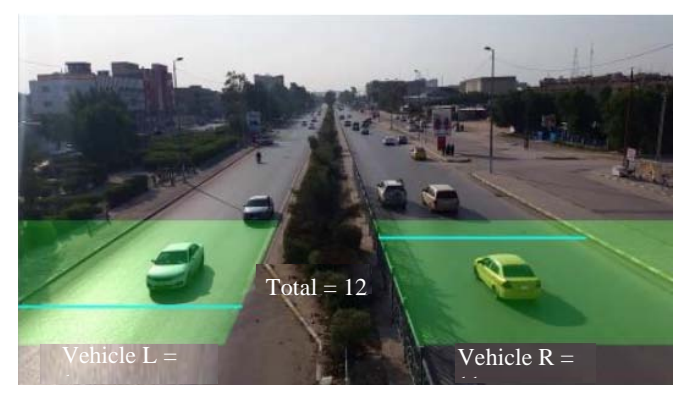

Fig. 6: Process of counting vehicles

$$
v=\frac{\mathrm{D}(\mathrm{km})}{\mathrm{T}(\mathrm{h})}(\mathrm{km} / \mathrm{h})
$$

The proposed system measures the elapsed time by recording the initial time $\left(\mathrm{t}_{1}\right)$ after detecting vehicle and recording the time $\left(\mathrm{t}_{2}\right)$ in the next image frame. Then, the elapsed time is the difference between them as shown in Eq. 3:

$$
\mathrm{t}=\mathrm{t}_{2}-\mathrm{t}_{1}(\text { sec })
$$

The distance can be measured by counting the Pixels (P) that the vehicle has moved. Then to convert the pixel number to real distance (d) in metre, we multiply by a Metre/Pixel ratio (MP-ratio) which has been calculated when the system has been calibrated. These parameters have ben demonstrated in Fig. 6. MP-ratio is determined by measuring the distance between two distinctive landmarks in metre and counting the pixel number between these landmarks in the image and then dividing these two values. Equation 4 shows how the distance has been calculated:

$$
\mathrm{d}=\mathrm{P} \times \mathrm{MP}-\operatorname{ratio}(\mathrm{m})
$$

The speed can be measured in Eq. 5. It is important to mention that the value (3.6) has been multiplied to convert units from $(\mathrm{m} / \mathrm{sec}$.) to $(\mathrm{km} / \mathrm{h})$ :

$$
v=\frac{\mathrm{d}}{\mathrm{t}} \times 3.6(\mathrm{~km} / \mathrm{h})
$$

To increase the accuracy of the speed estimation, the speed has been measured three times for each vehicle and the average has been taken as shown in Eq. 6:

$$
v_{\mathrm{av}}=\frac{v_{1}+v_{2}+v_{3}}{3}(\mathrm{~km} / \mathrm{h})
$$

\section{Experiments}

Testing the proposed method for vehicles counting: The proposed approach was tested using six data sets. Each data set contains a video with $2 \mathrm{~min}$ length recorded from a camera mounted on high place and directed to the road. These data sets were recorded in different places, different times and different weather conditions. The first two data sets were recorded in clear weather at about 12:30 pm (Fig. 7 which shows glimpse of the recorded videos). The second two data sets were recorded in a dusty weather at about 9:00 am. The last two data sets were recorded at night $(8: 00 \mathrm{pm})$. These data sets show high traffic videos. The results of the proposed counting algorithm were compared with a manual counting of vehicles in the recorded videos. The manual counting was considered as a ground truth.

Testing the proposed method for vehicle speed measurement: The vehicle speed measurement part of the proposed algorithm was tested using five videos from 5 datasets available online (Luvizon et al. 2017). These videos were recorded by 5-megapixel camera with resolution of $1920 \times 1080$ and 30.15 frames/sec. These videos are classified to 5 categories according to their recording and weather and situations (Fig. 8 shows samples of these data sets). These data sets have a ground truth file for the actual speed of each vehicle. The actual speed was recorded by a high precision speed meter (Luvizon et al., 2017). The measured speed by our proposed approach were compared with recorded ground truth values. 


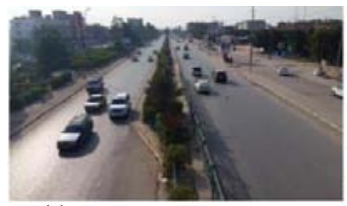

(c)
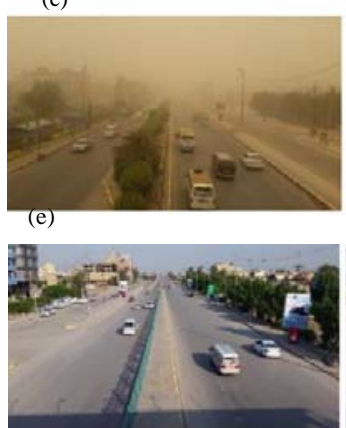

(b)
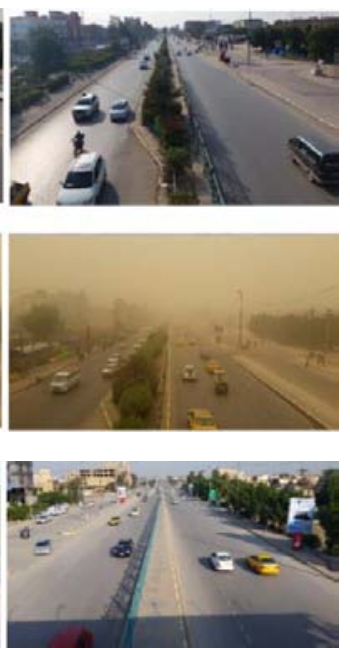

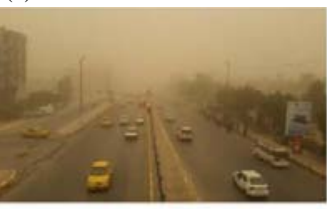

(d)
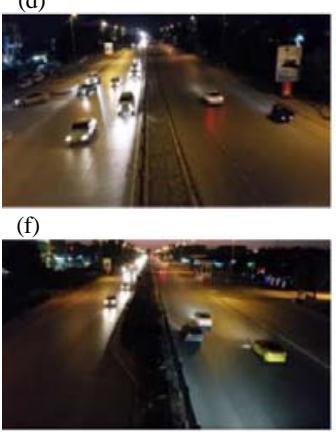
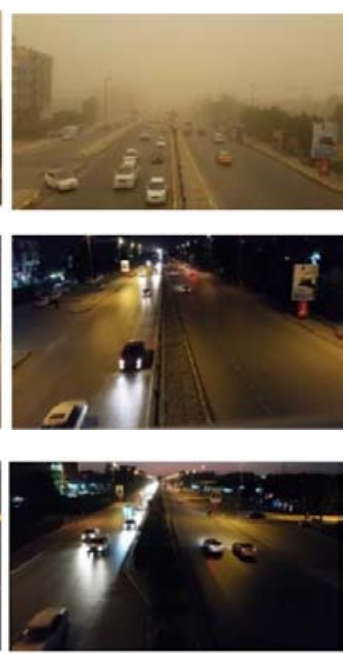

Fig. 7: Samples of the data sets used for vehicles counting evaluation: a) 1 st set; b) 2nd set; c) 3rd set; d) 4th set; e) 5th set and f) 6th set

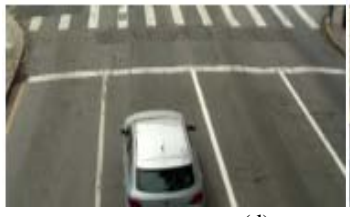

(d)

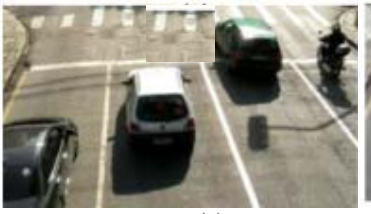

(e)

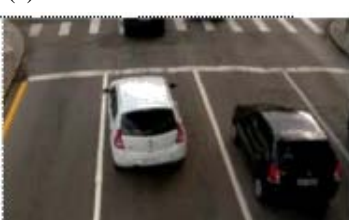

Fig. 8: Samples of the data sets used for speed measurement evaluation (Luvizon et al., 2017); a) 1st set; b) 2nd set; c) 3rd set; d) 4th set; e) 5th set and f) 6th set

Table 1: Evaluation result of the proposed algorithm for vehicle counting

\begin{tabular}{|c|c|c|c|c|c|c|c|c|c|}
\hline \multirow[b]{2}{*}{ Weather } & \multicolumn{2}{|c|}{ Recording info } & \multicolumn{2}{|c|}{ Manual counts } & \multirow[b]{2}{*}{ Total } & \multicolumn{3}{|c|}{ Proposed algorithm } & \multirow[b]{2}{*}{ Accuracy $\%$} \\
\hline & Local & Date & Left road & Right road & & Left road & Right road & Total & \\
\hline Clear-weather-1 & $2: 21 \mathrm{PM}$ & $30-11-2017$ & 64 & 68 & 132 & 72 & 65 & 137 & 96.21 \\
\hline Clear-weather-2 & $2: 23 \mathrm{PM}$ & $30-11-2017$ & 86 & 68 & 154 & 83 & 63 & 146 & 94.81 \\
\hline Dusty-weather-1 & $9: 26 \mathrm{AM}$ & $30-10-2017$ & 104 & 114 & 218 & 109 & 95 & 204 & 93.58 \\
\hline Dusty-weather-2 & $9: 29 \mathrm{AM}$ & $30-10-2017$ & 185 & 101 & 286 & 194 & 71 & 265 & 92.66 \\
\hline Night-time-1 & 5:32 PM & $01-12-2017$ & 88 & 92 & 180 & 98 & 108 & 206 & 85.56 \\
\hline Night-time-2 & 5:34 PM & $01-12-2017$ & 77 & 84 & 161 & 101 & 89 & 190 & 81.99 \\
\hline Average & -- & -- & -- & -- & -- & -- & -- & -- & 90.80 \\
\hline
\end{tabular}

\section{RESULTS AND DISCUSSION}

We tested the vehicle counting part of the proposed algorithm on 6 different data sets. Table 1 shows the evaluation results. In the first 2 data sets, the weather was clear in the footage and at the day time. The counting accuracy are 96.21 and $94.81 \%$, respectively. In the 3rd and 4th data sets, the counting accuracy are 93.58 and $92.66 \%$, respectively which is less than that in the previous two sets. This is because of the bad weather condition where the weather was dusty. However, the results still good and acceptable. In the 5th and 6th data sets, the counting accuracy are 85.56 and $81.99 \%$. The results are not good as in the previous sets, since, the 
data sets were recorded at night with low lighting. Moreover, the vehicles lights add more complexity to the situation. However, the results still acceptable. Overall,

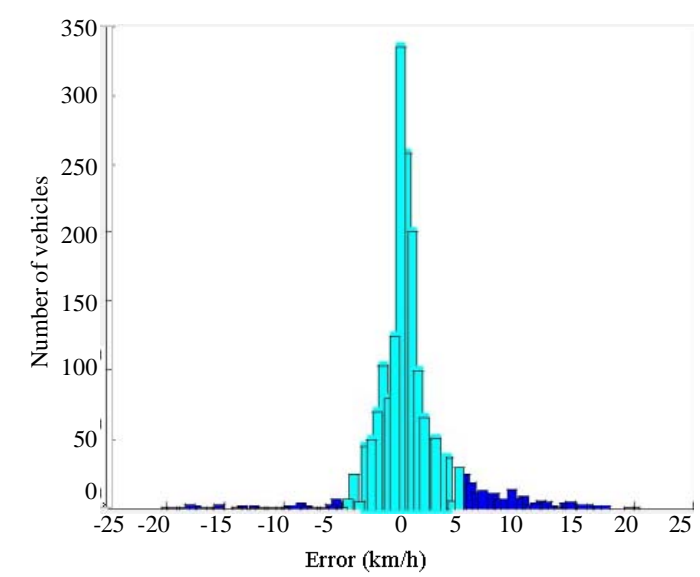

Fig. 9: The distribution of the speed measurement errors

(a)

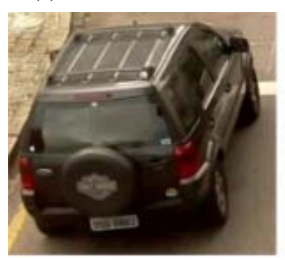

(e)

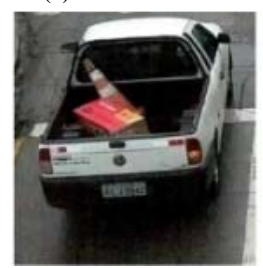

(i)

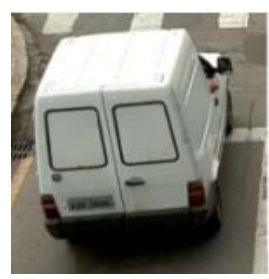

(b)

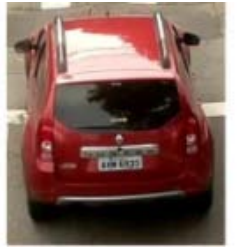

(f)

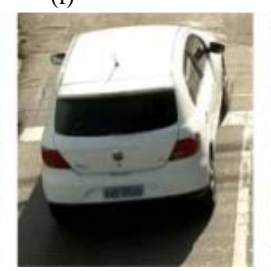

(j)

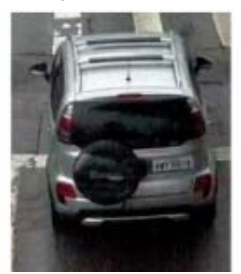

the proposed approach has shown very good overall counting accuracy with $90.8 \%$. We used the following accuracy relation Eq. 7 (Gupte et al., 2002) (Fig. 9 and 10):

Regarding the evaluation of speed measurement, we tested vehicle speed measurement part of the proposed algorithm on 5 videos from five datasets available (Luvizon et al., 2017). The speeds measured by our method were compared with the ground truth speeds obtained by the inductive loop detectors. The measurement was considered acceptable if it is within the $(-5,+5 \mathrm{~km} / \mathrm{h})$ error interval. It was found that $88.21 \%$ of the speeds measurements were within the acceptable error

(c)

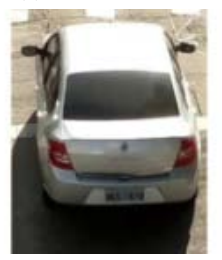

(g)

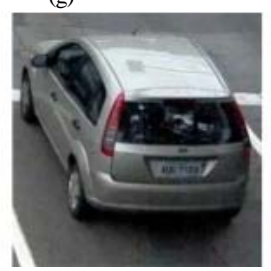

(k)

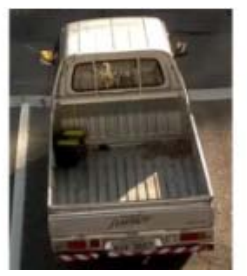

(d)

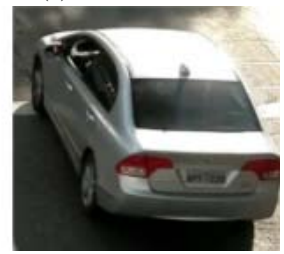

(h)

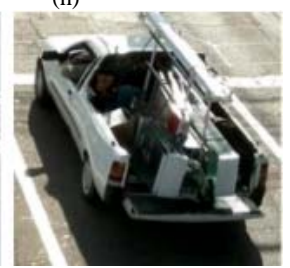

(1)

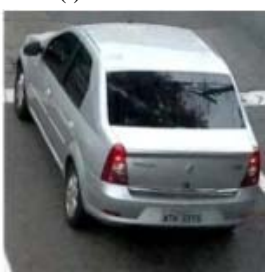

Fig. 10: Examples of evaluation results of the vehicles speeds measurements; a) Set01-video01 (Frame No.: 2287) Measured: $20.4 \mathrm{~km} / \mathrm{h}$ (Actual: $21.02 \mathrm{~km} / \mathrm{h}$ ); b) Set01-video01 (FrameNo.: 880) Measured: $50.08 \mathrm{~km} / \mathrm{h}$ (Actual: 50.84 $\mathrm{km} / \mathrm{h}$ ); c) Set02-video01 (Frame No.: 2020) Measured: $21.33 \mathrm{~km} / \mathrm{h}$ (Actual: $23.37 \mathrm{~km} / \mathrm{h}$ ); d) Set05-video01 (Frame No.: 36118) Measured: $45.79 \mathrm{~km} / \mathrm{h}$ (Actual: $48.07 \mathrm{~km} / \mathrm{h}$ ); e) Set04-video01 (Frame No.: 10853) Measured: $22.44 \mathrm{~km} / \mathrm{h}$ (Actual:22.37 km/h); f) Set02-video01 (Frame N.: 7332) Measured: $50.73 \mathrm{~km} / \mathrm{h}$ (Actual: $50.71 \mathrm{~km} / \mathrm{h}$ ); g) Set03video01 (Frame No.: 1914) Measured: 33.39 km/h (Actual: 33.94 km/h); h) Set02-video01 (Frame No.: 534 ) Measured: $45.45 \mathrm{~km} / \mathrm{h}$ (Actual: $43.57 \mathrm{~km} / \mathrm{h}$ ); i) Set01-video01 (Frame No.: 767) Measured: $48.04 \mathrm{~km} / \mathrm{h}$ (Actual: 50.59 km/h); j) Set04-video01 (FrameNo.: 904) Measured: $47.61 \mathrm{~km} / \mathrm{h}$ (Actual:50.63 km/h); k) Set05-video01 (FrameNo.: 20519) Measured: $50.08 \mathrm{~km} / \mathrm{h}$ (Actual: $49.06 \mathrm{~km} / \mathrm{h}$ ) and 1) Set03-video01 (Frame No.: 2047) Measured: $44.52 \mathrm{~km} / \mathrm{h}$ (Actual: $46.89 \mathrm{~km} / \mathrm{h}$ ) 
interval. Whereas the ratio of the speeds measurements lower and above the acceptable error interval were 2.36 and $9.43 \%$, respectively. Figure 9 shows the distribution of the measurement errors. It is important to mention that these ratios were calculated based on the valid vehicles that have speed measurement in the ground truth. Figure 10 shows examples of measured speeds.

\section{CONCLUSION}

This study addressed the problem of vehicles counting and speed estimation using camera mounted on the road. We proposed an algorithm that consists from motion detection, vehicles detection, speed measurements and counting. Motion detection is done by subtracting two successive frames. After that vehicles are detected by threshold, morphological and contour operations. Finally, after obtaining vehicles in the region of the interest, vehicles can be counted and their speed can be measured. To evaluate the proposed algorithm, testing vehicle counting and speed measurement were done separately. The vehicle counting part of the proposed algorithm was tested using six data sets. The data sets contain different times and weather conditions. The vehicle counting algorithm is compared with the manual counting which is considered as ground truth. The experiments show a good result as $90.8 \%$ for vehicle counting.

\section{RECOMMENDATIONS}

To evaluate the proposed vehicle speed measurement algorithm, we used five videos from 5 datasets available online where these data sets have a ground truth file for the actual speed of each vehicle using very precise inductive loop detector. Experiments shows that the speeds measurements were within the acceptable error interval where the speed measurement was considered acceptable if it is within the $(-5,+5 \mathrm{~km} / \mathrm{h})$.

\section{REFERENCES}

Biswas, D., H. Su, C. Wang, J. Blankenship and A. Stevanovic, 2017. An automatic car counting system using overfeat framework. Sens., 17: 1-13.

Buch, N., S.A. Velastin and J. Orwell, 2011. A review of computer vision techniques for the analysis of urban traffic. IEEE. Trans. Intell. Transp. Syst., 12: 920-939.

Chen, S.C., M.L. Shyu and C. Zhang, 2001. An intelligent framework for spatio-temporal vehicle tracking. Proceedings of the 2001 IEEE International Conference on Intelligent Transportation Systems (Cat. No.01 TH8585), August 25-29, 2001, IEEE, Oakland, California, ISBN:0-7803-7194-1, pp: 213-218.
Czajewski, W. and M. Iwanowski, 2010. Vision-based vehicle speed measurement method. Proceedings of the International Conference on Computer Vision and Graphics, September 20-22, 2010, Springer, Berlin, Heidelberg, Germany, ISBN:978-3-642-15909-1, pp: 308-315.

Daigavane, P.M. and P.R. Bajaj, 2010. Real Time Vehicle Detection and Counting Method for Unsupervised Traffic Video on Highways Unsupervised Traffic Video on Highways. Intl. J. Comput. Sci. Network Secur., 10: 112-117.

Daniel, D.J. and S. Pumrin, 2000. An algorithm to estimate mean traffic speed using uncalibrated cameras. IEEE Trans. Intel. Transport. Syst., 1: 98-107.

Dogan, S., M.S. Temiz and S. Kulur, 2010. Real time speed estimation of moving vehicles from side view images from an uncalibrated video camera. Sens., 10: 48054824.

Fuchs, H., M.A. Livingston, R. Raskar, K. Keller and J.R. Crawford et al., 1998. Augmented reality visualization for laparoscopic surgery. Proceedings of the International Conference on Medical Image Computing and Computer-Assisted Intervention, October 11-13, 1998, Springer, Berlin, Heidelberg, Germany, ISBN: 978-3-540-65136-9, pp: 934-943.

Fujii, K., G. Gras, A. Salerno and G.Z. Yang, 2018. Gaze gesture based human robot interaction for laparoscopic surgery. Med. Image Anal., 44: 196-214.

Gao, T., Z.G. Liu, W.C. Gao and J. Zhang, 2009. Moving Vehicle Tracking based on SIFT Active Particle Choosing. In: Advances in Neuro-Information Processing, Koppen, M., N. Kasabov and G. Coghill (Eds.). Springer, Berlin, Heidelberg, ISBN: 978-3-64203039-0, pp: 695-702.

Garg, M. and S. Goel, 2013. Real-time license plate recognition and speed estimation from video sequences. ITSI. Trans. Electr. Electron. Eng., 1: 1-4.

Garibotto, G., P. Castello, E.D. Ninno, P. Pedrazzi and Z. Zan, 2001. Speed-vision: Speed measurement by license plate reading and tracking. Proceedings of the IEEE Conference on Intelligent Transportation Systems, August 25-29, 2001, IEEE, Piscataway, New Jersey, USA., pp: 585-590.

Gupte, S., O. Masoud, R.F.K. Martin and N.P. Papanikolopoulos, 2002. Detection and Classification of vehicles. IEEE Trans. Intelligent Transport. Syst., 3: 37-47.

He, X.C. and N.H.C. Yung, 2007. A novel algorithm for estimating vehicle speed from two consecutive images. Proceedings of the 2007 IEEE Workshop on Applications of Computer Vision (WACV '07), February 21-22, 2007, IEEE, Austin, Texas, ISBN:07695-2794-9, pp: 12-12. 
Jun, G., J.K. Aggarwal and M. Gokmen, 2008. Tracking and segmentation of highway vehicles in cluttered and crowded scenes. Proceedings of the IEEE Workshop on Applications of Computer Vision WACV 2008, January 7-9, 2008, IEEE, Copper Mountain, Colorado, ISBN:978-1-4244-1913-5, pp: 1-6.

Liang, M., X. Huang, C.H. Chen, X. Chen and A.O. Tokuta, 2015. Counting and classification of highway vehicles by regression analysis. IEEE. Trans. Intell. Transp. Syst., 16: 2878-2888.

Luvizon, D.C., B.T. Nassu and R. Minetto, 2017. A videobased system for vehicle speed measurement in urban roadways. IEEE. Trans. Intell. Transp. Syst., 18: 1393-1404.

Ma, X. and W.E.L. Grimson, 2005. Edge-based rich representation for vehicle classification. Procedings of the 10th IEEE International Conference on Computer Vision (ICCV'05), October 17-21, 2005, IEEE, Beijing, China, ISBN:0-7695-2334-X, pp: 11851192.

Madasu, V.K. and M. Hanmandlu, 2010. Estimation of vehicle speed by motion tracking on image sequences. Proceedings of the 2010 IEEE Symposium on Intelligent Vehicles, June 21-24, 2010, IEEE, San Diego, California, ISBN:978-1-4244-7866-8, pp: 185190 .

Maduro, C., K. Batista, P. Peixoto and J. Batista, 2008. Estimation of vehicle velocity and traffic intensity using rectified images. Proceedings of the 200815 th IEEE International Conference on Image Processing, October 12-15, 2008, IEEE, San Diego, California, ISBN:978-1-4244-1765-0, pp: 777-780.

Matsuno, F. and S. Tadokoro, 2004. Rescue robots and systems in Japan. Proceedings of the 2004 IEEE International Conference on Robotics and Biomimetics, August 22-26, 2004, IEEE, Shenyang, China, ISBN:0-7803-8614-8, pp: 12-20.
McCall, J.C. and M.M. Trivedi, 2006. Video-based lane estimation and tracking for driver assistance: Survey, system, and evaluation. Intell. Transport. Syst. IEEE Transact., $7: 20-37$.

Palaio, H., C. Maduro, K. Batista and J. Batista, 2009. Ground plane velocity estimation embedding rectification on a particle filter multi-target tracking. Proceedings of the 2009 IEEE International Conference on Robotics and Automation, May 12-17, 2009, IEEE, Kobe, Japan, ISBN:978-1-4244-2788-8, pp: $825-830$.

Salvi, G., 2012. An automated vehicle counting system based on blob analysis for traffic surveillance. Proceedings of the International Conference on Image Processing, Computer Vision and Pattern Recognition(IPCV), July 16-1 12, 2012, CSREA Press, Las Vegas, Nevada, USA., ISBN:9781601322258, pp: 397-402.

Taylor, R.H., A. Menciassi, G. Fichtinger, P. Fiorini and P. Dario, 2016. Medical Robotics and ComputerIntegrated Surgery. In: Springer Handbook of Robotics, Siciliano, B. and O. Khatib (Eds.). Springer, Cham, Switzerland, ISBN:978-3-319-32550-7,pp: 16571684.

Wang, X., 2012. Intelligent multi-camera video surveillance: A review. Pattern Recognit. Lett., 34: 39.

Zhiwei, H., L. Yuanyuan and Y. Xueyi, 2007. Models of vehicle speeds measurement with a single camera. Proceedings of the 2007 International Conference on Computational Intelligence and Security Workshops (CISW 2007), December 15-19, 2007, IEEE, Heilongjiang, China, ISBN:0-7695-3073-7, pp: 283-286. 